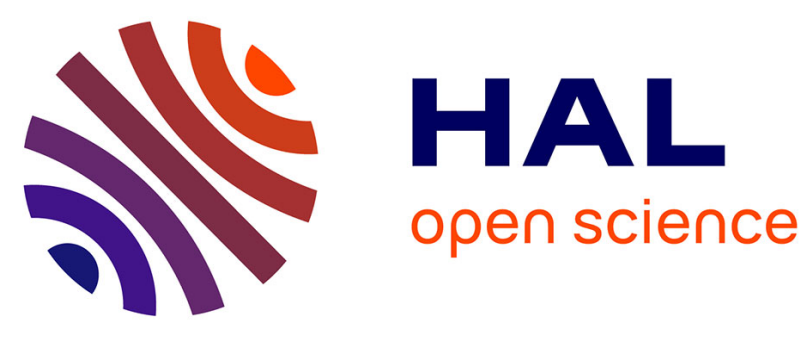

\title{
Déterminisme de la phénologie des forêts tempérées françaises : étude sur les peuplements du réseau Renecofor
}

François Lebourgeois, Jean-Claude Pierrat, Vincent Perez, Christian Piedallu, Sébastien Cecchini, Erwin Ulrich

\section{To cite this version:}

François Lebourgeois, Jean-Claude Pierrat, Vincent Perez, Christian Piedallu, Sébastien Cecchini, et al.. Déterminisme de la phénologie des forêts tempérées françaises: étude sur les peuplements du réseau Renecofor. Revue forestière française, 2008, 60 (3), pp.323-343. 10.4267/2042/19767. hal-03449805

\section{HAL Id: hal-03449805 \\ https://hal.science/hal-03449805}

Submitted on 25 Nov 2021

HAL is a multi-disciplinary open access archive for the deposit and dissemination of scientific research documents, whether they are published or not. The documents may come from teaching and research institutions in France or abroad, or from public or private research centers.
L'archive ouverte pluridisciplinaire HAL, est destinée au dépôt et à la diffusion de documents scientifiques de niveau recherche, publiés ou non, émanant des établissements d'enseignement et de recherche français ou étrangers, des laboratoires publics ou privés. 


\title{
BIOLOGIE ET ECOLOGIE
}

\section{DÉTERMINISME DE LA PHÉNOLOGIE DES FORÊTS TEMPÉRÉES FRANÇAISES : ÉTUDE SUR LES PEUPLEMENTS DU RÉSEAU RENECOFOR}

\author{
François Lebourgeois - Jean-Claude Pierrat - Vincent Perez \\ Christian Piedallu - Sébastien Cecchini - Erwin Ulrich
}

La phénologie des plantes peut être définie comme l'étude des cycles annuels de développement et de leur déterminisme. Le rythme d'apparition de ces différentes phases dépend de facteurs aussi bien biologique que physique, comme l'espèce, la latitude, les conditions édaphique ou topo-climatique (Ahas et al., 2000 ; Dittmar et Elling, 2006 ; Richardson et al., 2006 ; Wielgolaski, 2001). Étant sous fort déterminisme environnemental, les observations phénologiques sont parmi les indicateurs les plus pertinents pour juger de la manière dont les espèces répondent à leur milieu. Ainsi, cette discipline a émergé récemment comme un sujet majeur dans les recherches en écologie (Estrella et al., 2007 ; Kramer et al., 2000 ; Parmesan, 2007 ; Pudas et al., 2007 ; Schwartz, 1999 ; Sparks et Menzel, 2002 ; Walther et al., 2002), particulièrement pour mettre en évidence les changements de saison de végétation ou de stockage de carbone en relation avec le réchauffement observé depuis les dernières décennies (Ahas et al., 2002 ; Beaubien et Freeland, 2000 ; Chen et al., 2005 ; Chmielewski et Rötzer, 2001 ; Chmielewski et al., 2004 ; Churkina et al., 2005 ; Linderholm, 2006 ; Lu et al., 2006 ; Menzel, 2000 ; Menzel et Fabian, 1999 ; Menzel et al., 2006 ; Murray et al., 1989 ; Schwartz et al., 2006 ; Tao et al., 2006). Dans la plupart de ces travaux menés essentiellement en Europe centrale et de l'Est, ce sont des données historiques issues de réseaux phénologiques qui ont été utilisées (Menzel et al., 2006). Afin de pouvoir mener de tels travaux en France, une base de données regroupant les observations disponibles sur les espèces végétales est en cours de création (Groupe de recherche, système d'information phénologique pour la gestion et l'étude des changements climatiques, http://gdr.obs-saisons.fr/). Actuellement, ce sont les données issues des observations menées depuis 1997 dans le réseau RENECOFOR qui correspondent aux séries homogènes les plus longues disponibles en France pour les peuplements forestiers adultes (Ulrich, 1995). Dans un article publié en 2002 dans la Revue forestière française (Lebourgeois et al., 2002), nous avons présenté les premiers résultats sur les observations phénologiques du réseau pour la période 1997-1999. Celles-ci ayant été maintenues depuis, nous disposons maintenant d'observations sur la période 1997-2006 pour la majorité des 102 peuplements. Si cette période apparaît encore trop courte pour détecter des changements (aucune tendance n'a d'ailleurs été observée), une nouvelle analyse doit permettre de mieux quantifier la variabilité spatiale et temporelle des cycles de développement des espèces, et surtout de mieux appréhender le déterminisme climatique et géographique des différentes phases considérées.

Le premier objectif de cet article est donc de décrire la variabilité spatiale et temporelle de la phénologie de peuplements forestiers feuillus et résineux adultes en utilisant les dates de débourrement et de jaunissement observées sur la période 1997-2006. Dans un second temps, le déter- 
François Lebourgeois - Jean-Claude Pierrat - Vincent Perez - Christian Piedallu - Sébastien Cecchini - Erwin Ulrich

minisme des cycles annuels de développement est analysé à travers la prise en compte à la fois de données géographiques (latitude, longitude, altitude) et climatiques (températures, précipitations, évapotranspiration, bilan hydrique). Les modèles obtenus sont ensuite utilisés pour prédire les dates des phénophases et les prédictions sont cartographiées sur l'ensemble du territoire.

\section{MATÉRIEL ET MÉTHODES}

Les observations ont été effectuées sur 101 des 102 peuplements du réseau RENECOFOR et sur deux hêtraies luxembourgeoises qui sont soumises aux mêmes protocoles d'observations que les peuplements français. Les caractéristiques dendrométriques et stationnelles de ces 103 peuplements ont été largement présentées dans les nombreuses publications (rapports disponibles sur http://www.onf.fr/pro/Renecofor/publi.htm) et ne seront donc pas reprises ici. Les Chênes sessile et pédonculé correspondent à des peuplements de plaine du Nord de la France avec une répartition assez homogène de l'ouest à l'est (tableau I, ci-dessous). Les hêtraies sont localisées dans les plaines du Nord-Est et dans différents contextes montagnards du Sud et Sud-Est (altitude moyenne $1031 \mathrm{~m}$ ). L’Épicéa, le Sapin, et le Mélèze constituent des peuplements d'altitude dans la partie Est de la France. Enfin, pour les Pins et le Douglas, les localisations géographiques et les conditions écologiques sont plus variées. Hêtre, Chênes, Sapin, Mélèze et une partie des Épicéas ont des origines locales et correspondent à des peuplements régénérés naturellement. Les pineraies et les douglasaies sont généralement issues de plantation. Même si nous ne possédons aucune information précise quant à l'origine génétique des graines ou plants, la pratique forestière courante est de sélectionner des graines bien adaptées pour chaque contexte climatique, ce qui réduit le risque de biais dans les analyses.

\begin{tabular}{|c|c|c|c|c|c|}
\hline Espèces & $\begin{array}{l}\text { Nb sites/ } \\
\text { arbres }\end{array}$ & $\begin{array}{l}\text { Latitude }\left({ }^{\circ}\right) \\
\text { moyenne }\end{array}$ & $\begin{array}{l}\text { Longitude }\left(^{\circ}\right) \\
\text { moyenne }\end{array}$ & Âge & Dia $(\mathrm{cm})$ \\
\hline \multicolumn{6}{|l|}{ Feuillus } \\
\hline Fagus sylvatica .................. & $22 / 792$ & $46,8^{\circ}$ & $3,9^{\circ}$ & $97[41-161]$ & $40[25-50]$ \\
\hline Quercus petraea.................... & $21 / 756$ & $47,8^{\circ}$ & $3,6^{\circ}$ & $94[55-140]$ & $41[30-53]$ \\
\hline 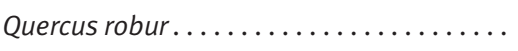 & $8 / 288$ & $47,1^{\circ}$ & $3,2^{\circ}$ & $71[36-134]$ & $38[20-56]$ \\
\hline \multicolumn{6}{|l|}{ Résineux } \\
\hline Abies alba ..... & $11 / 396$ & $45,5^{\circ}$ & $5,0^{\circ}$ & $94[42-169]$ & $49[39-58]$ \\
\hline Larix decidua . ...................... & $1 / 36$ & $44,7^{\circ}$ & $6,6^{\circ}$ & 132 & 40 \\
\hline Picea abies ...................... & $11 / 396$ & $46,2^{\circ}$ & $4,7^{\circ}$ & $66[24-182]$ & $40[24-56]$ \\
\hline Pinus nigra ssp. laricio var corsicana ........ & $2 / 72$ & $45,0^{\circ}$ & $5,5^{\circ}$ & $46-174$ & $36-66$ \\
\hline Pinus pinaster. .................... & $7 / 252$ & $44,9^{\circ}$ & $2,1^{\circ}$ & $31[15-62]$ & $29[21-44]$ \\
\hline Pinus sylvestris . . . . . . . . . . . . . . . & $14 / 504$ & $47,6^{\circ}$ & $3,6^{\circ}$ & $57[39-94]$ & $36[29-41]$ \\
\hline \multirow[t]{2}{*}{ Pseudotsuga menziesii ............... } & $6 / 216$ & $45,7^{\circ}$ & $2,2^{\circ}$ & $29[21-48]$ & $37[26-60]$ \\
\hline & $103 / 3708$ & & & 77 & 39 \\
\hline
\end{tabular}


Les observations ont concerné le débourrement et le jaunissement. Entre 1997 et 2006, 78 peuplements ont été observés sur au moins 8 ans et 42 sur l'ensemble de la période. Ainsi, sur les 10 années d'observations, 838 et 449 observations sont disponibles pour chacune des phases. Les observations ont été faites par les responsables locaux des placettes à l'aide de jumelles dans plus de $70 \%$ des cas. Chaque année, c'est la date à laquelle un certain pourcentage des 36 arbres "observation" du peuplement a présenté un certain stade de développement qui a été notée (Lebourgeois et al., 2002). Deux dates ont été retenues pour le débourrement. La première ( $\left.\mathrm{dd}_{1}\right)$ correspond à la date pour laquelle $10 \%$ des arbres ont présenté au moins $20 \%$ de bourgeons ouverts : jeunes feuilles visibles mais non complètement développées. La seconde (dd2) correspond au moment où $90 \%$ des arbres ont atteint ce même stade. La différence entre les deux dates donne une indication sur la durée du débourrement. Le même principe a été adopté pour les notations de jaunissement en fin de saison pour les feuillus et Mélèze. Les dates correspondent au moment où $10 \%$ et $90 \%$ des arbres ont présenté sur au moins $20 \%$ du houppier des feuilles jaunes (jj1 et jj2). Toutes les dates ont été exprimées en jours juliens c'est-à-dire en jours depuis le premier janvier (1 à 365 ou 366). Pour les feuillus, 4 durées de saison de végétation exprimées en jours ont été calculées à partir des 4 dates des deux phénophases.

Pour l'étude du déterminisme des phénophases, un total de 143 séries climatiques a été utilisé. Les données proviennent des 20 stations du sous-réseau météorologique lié aux placettes RENECOFOR et de 123 stations du réseau national Météo-France. Ces dernières ont été sélectionnées sur des critères de proximité et de représentativité par rapport aux peuplements forestiers (même altitude, pente, exposition...). La distance entre les sites et les stations a été en moyenne de $11,1 \mathrm{~km}$. Les moyennes des températures minimales, maximales, moyennes mensuelles et des précipitations ont été calculées sur la période 1997-2006. Ces données élémen-

taires ont été complétées par des calculs d'évapotranspiration selon les formules de Turc et Thornthwaite (Lebourgeois et Piedallu, 2005) et de bilan hydrique climatique (P - ETP). Pour chaque site, la latitude, la longitude, l'altitude et les distances à l'océan le plus proche ou à la Méditerranée ont également été intégrées comme variables explicatives. En effet, même si les variations de ces facteurs géographiques expriment en grande partie des variations du régime thermique et, dans une moindre mesure pluviométrique, déjà prises en compte par les données climatiques, de nombreuses études ont montré qu'elles amélioraient notablement les niveaux d'explication ou de prédiction des modèles (Chmielewski et Rötzer, 2001; Rötzer et Chmielewski, 2001).

TABLEAU I

Caractéristiques moyennes des 103 peuplements échantillonnés dans le réseau RENECOFOR. Âge en 1994. Dia : diamètre à 1,30 m. Les valeurs entre crochets indiquent la gamme de variation du paramètre 
François Lebourgeois - Jean-Claude Pierrat - Vincent Perez - Christian Piedallu - Sébastien Cecchini - Erwin Ulrich

Les analyses statistiques ont fait appel à des techniques de régression multivariables connues sous le nom de "Forêts d'arbres aléatoires » (RandomForests, RF) (Berk, 2006 ; Breiman, 1996, 2001a, b ; Li, 2006 ; Liaw et Wiener, 2002 ; Peters et al., 2007 ; Verbyla, 1987). L'intérêt de ces méthodes ne cesse d'augmenter depuis les dernières années dans les recherches en écologie car elles fournissent des prédictions plus stables et sans biais de sélection par rapport aux régressions multiples pas-à-pas (Garzon et al., 2006 ; Gislason et al., 2006 ; Iverson et Prasad, 1998 ; Iverson et al., 1999 ; Peters et al., 2007 ; Prasad et al., 2006 ; Remm, 2004 ; Vayssières et al., 2000 ; Waheed et al., 2006). L'objectif est toujours d'expliquer la variabilité d'un facteur (ici les dates des phénophases) à partir de facteurs écologiques multiples et de définir lesquels expliquent le mieux le déterminisme du facteur étudié. Nous ne présenterons pas ici ces analyses en détail et renvoyons les lecteurs aux nombreuses publications de l'inventeur de cette méthode, Léo Breiman, ainsi qu'à la note méthodologique en fin de l'article (p. 339). In fine, différentes valeurs statistiques permettent de juger de la qualité d'ajustement, d'explication et de prédiction des modèles.

TABLEAU II

$$
\begin{gathered}
\text { Dates moyennes (écart-type) [minimum-maximum] } \\
\text { de débourrement (dd1 et dd2) et de jaunissement (jj1 et jj2) } \\
\text { pour chaque espèce (période 1997-2006) } \\
\text { Les données sont exprimées en jour julien (1 } 1 \text { er mai = jj : 121) } \\
n=\text { nombre d'observations disponibles }
\end{gathered}
$$

\begin{tabular}{|c|c|c|c|c|}
\hline \multirow{2}{*}{ Espèces } & \multicolumn{4}{|c|}{ Débourrement } \\
\hline & $\mathrm{n}$ & dd1 & $\mathrm{dd} 2$ & durée (jours) \\
\hline \multicolumn{5}{|l|}{ Feuillus } \\
\hline Fagus sylvatica .............. & 178 & $112(12,3)[71-181]$ & $121(10,0)[98-188]$ & $9,0(5,4)$ \\
\hline Quercus petraea ............. & 182 & $100(10,2)[78-124]$ & $111(12,3)[88-195]$ & $11,1(6,8)$ \\
\hline 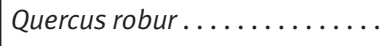 & 71 & $100(13,0)[71-125]$ & $112(14,7)[78-137]$ & $12,9(9,7)$ \\
\hline \multicolumn{5}{|l|}{ Quercus petraea............. } \\
\hline nord-est & 82 & $105(8,9)[83-124]$ & 115 (10) [90-153] & $10,9(7,7)$ \\
\hline nord-ouest & 100 & $95(9,1)[78-114]$ & $107(12,7)[88-195]$ & $11,4(6,0)$ \\
\hline \multicolumn{5}{|l|}{ Quercus robur.............. } \\
\hline nord-est & 37 & $109(6,6)[97-125]$ & $117(6,5)[97-132]$ & $8,9(4,8)$ \\
\hline nord-ouest & 34 & $89(9,8)[71-108]$ & $107(18,8)[78-137]$ & $16,9(11,6)$ \\
\hline \multicolumn{5}{|l|}{ Conifères } \\
\hline 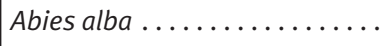 & 98 & $133(12)[102-172]$ & $141(11,9)[116-179]$ & $9,0(4,2)$ \\
\hline Larix decidua................ & 10 & $130(5)[122-138]$ & $141(6,2)[129-146]$ & $10,5(4,9)$ \\
\hline Picea abies ................ & 83 & $132(11,5)[97-166]$ & $142(11,3)[114-173]$ & $10,8(6,3)$ \\
\hline Pinus nigra ssp. Laricio ........ & 14 & $121(14,9)[104-159]$ & $131(14,7)[114-166]$ & $10,2(4,1)$ \\
\hline 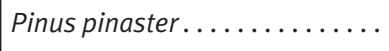 & 46 & $115(17,8)[80-156]$ & $126(16,4)[97-163]$ & $11,6(6,6)$ \\
\hline Pinus sylvestris . ............... & 108 & $125(12)[90-159]$ & $135(12,3)\left[104^{-166}\right]$ & $10,9(5,8)$ \\
\hline Pseudotsuga menziesii ....... & 48 & $124(11,9)[97-142]$ & $136(11,6)[111-161]$ & $12,1(6,2)$ \\
\hline
\end{tabular}

La durée de la saison de végétation donnée dans ce tableau correspond à la différence entre jj2 et dd1 (voir texte pour les détails).

Pour les chênaies, les données du Nord-Est et du Nord-Ouest sont présentées séparément. 


\begin{tabular}{|c|c|c|c|c|c|}
\hline \multirow[b]{2}{*}{ Espèces } & \multicolumn{4}{|c|}{ Jaunissement } & \multirow{2}{*}{$\begin{array}{l}\text { Durée } \\
\text { de la saison } \\
\text { de végétation } \\
\text { (jours) }\end{array}$} \\
\hline & $n$ & $\mathrm{jj} 1$ & ji2 & $\begin{array}{l}\text { durée } \\
\text { (jours) }\end{array}$ & \\
\hline \multicolumn{6}{|l|}{ Feuillus } \\
\hline Fagus sylvatica ....... & 179 & $275(16,5)[223-318]$ & $292(16)$ [244-332] & $16,8(10,3)$ & $180(22)[112-245]$ \\
\hline Quercus petraea ..... & 188 & $284(14,2)[247-323]$ & $300(11,3)[264-334]$ & $15,8(9,1)$ & $200(15,5)[154-245]$ \\
\hline Quercus robur....... & 72 & $283(15,8)[251-332]$ & $300(14,6)[272-345]$ & $16,6(8,4)$ & $200(25,4)[161-266]$ \\
\hline \multicolumn{6}{|l|}{ Quercus petraea..... } \\
\hline nord-est & 87 & $283(14,7)[247-317]$ & $298(11,9)[264-325]$ & $14,8(9,2)$ & $192(14,8)[154-231]$ \\
\hline nord-ouest & 101 & $286(13,6)[248-323]$ & $302(10,5)[271-334]$ & $16,6(9)$ & $207(12,9)[182-245]$ \\
\hline \multicolumn{6}{|l|}{ Quercus robur........ } \\
\hline nord-est & 35 & $278(10,9)[258-302]$ & $292(10,1)[272-316]$ & $13,4(7,5)$ & $181(10,8)[161-217]$ \\
\hline nord-ouest & 37 & $288(18,1)[251-332]$ & $307(14,1)[285-354]$ & $19,5(8,2)$ & $220(20,8)[189-266]$ \\
\hline Conifères & & & & & \\
\hline Larix decidua. & 10 & $273(4,7)[264-282]$ & $288(5,0)[278-293]$ & $14,7(2,2)$ & $158(7,6)[147-168]$ \\
\hline
\end{tabular}

\section{RÉSULTATS}

\section{Variabilité interspécifique et spatiale}

Entre 1997 et 2006, la date de débourrement a varié du 12 mars au 30 juin avec un écart-type entre toutes les observations de 17,3 jours. Pour les chênaies, le débourrement commence en moyenne vers la mi-avril (tableau II, pp. 326-327 et figure 1, p. 328). L'amplitude maximale observée entre la date la plus précoce et la date la plus tardive a été de 46 jours pour le Chêne sessile et 54 jours pour le Chêne pédonculé. Pour chacune des chênaies, la variabilité moyenne entre les années a été de 4 à 6 jours avec des écarts extrêmes de 11 à 36 jours (moyenne 21 jours). En moyenne, le débourrement dure 12 jours. Il est également apparu que les chênaies de l'Ouest de la France débourrent en général 10 à 20 jours plus tôt que les peuplements situés dans l'Est (tableau II, pp. 326-327 et figure 1, p. 328). Le débourrement est décalé d'environ 2 jours par degré de longitude (figure 2, p. 329). Le jaunissement apparaît vers la mi-octobre. Le début de cette phase présente une variabilité entre année plus grande que celle observée pour le débourrement (15 jours en moyenne) et varie également selon la localisation géographique. Ainsi, la longueur de la saison de végétation apparaît plus courte dans l’Est ( 200 jours) en conséquence d'un débourrement plus tardif et d'un jaunissement plus précoce.

Pour le Hêtre, la saison de végétation débute plus tard vers la fin de la troisième semaine d'avril et la mise en place des feuilles dure environ 9 jours (tableau II, pp. 326-327 et figure 1, p. 328). Dans un même peuplement, la variabilité entre les années et l'écart moyen des extrêmes sont du même ordre que ceux observés pour les chênaies (respectivement 4 à 8 jours et 21 jours). Le jaunissement des feuilles intervenant début octobre, la saison de végétation est en moyenne de 180 jours, c'est-à-dire réduite d'environ 20 jours par rapport aux chênaies. Étant donné que les hêtraies sont localisées en majorité dans la grande partie Est de la France, le gradient longitudinal n'est pas significatif (figure 2, p. 329). 
François Lebourgeois - Jean-Claude Pierrat - Vincent Perez - Christian Piedallu - Sébastien Cecchini - Erwin Ulrich

FIGURE 1

DATES MOYENNES DE DÉBOURREMENT ENTRE 1997 ET 2006

(dd1 exprimée en jours juliens)

Seules les 8 espèces les plus représentées ont été cartographiées.

Plus le rond est grand, plus la date de débourrement est tardive.

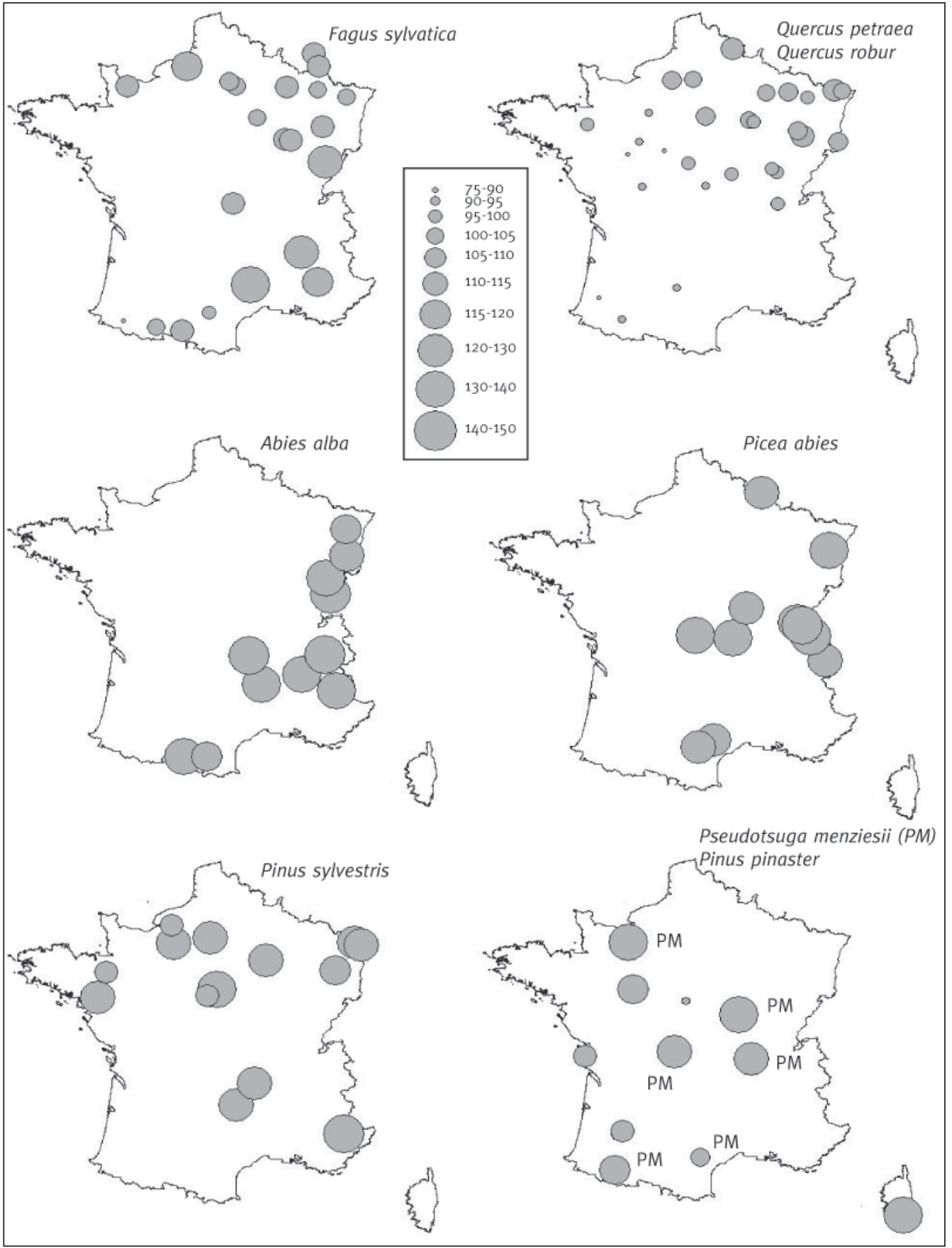



DU RÉSEAU RENECOFOR

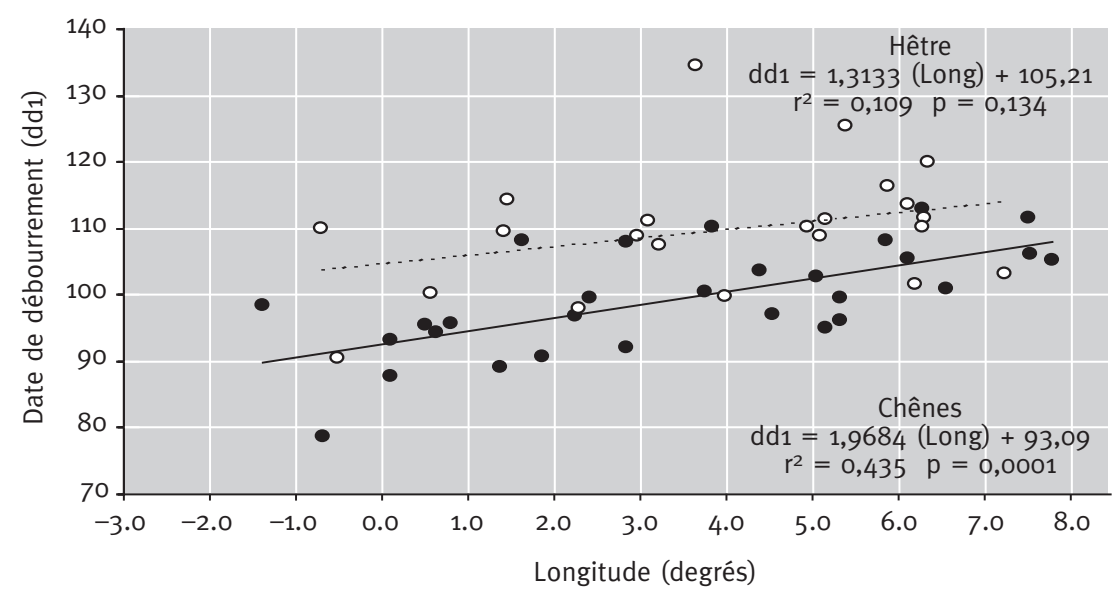

Le débourrement est plus tardif pour les résineux, mais les variabilités entre années, les écarts extrêmes au sein de chaque peuplement et la durée de mise en place des feuilles sont comparables à ceux observés pour les feuillus (tableau II, pp. 326-327). L'altitude étant très variable pour les conifères, la date de débourrement varie fortement selon ce paramètre avec un retard moyen d'environ 1,5 jour par $100 \mathrm{~m}$ (figure 3, ci-dessous). Si l'on considère chaque espèce séparément, le gradient varie de 1,1 à 1,8 jour par $100 \mathrm{~m}$. Cet effet altitudinal exprime en grande partie la diminution de la température en contexte montagnard qui correspond à une diminution moyenne de $0,5{ }^{\circ} \mathrm{C}$ de la température moyenne annuelle par $100 \mathrm{~m}$ d'élévation.

\section{FIGURE 3 RELATION ENTRE LA DATE MOYENNE DE DÉBOURREMENT (dd1, 1997-2006)} ET L'ALTITUDE POUR LES PEUPLEMENTS DE RÉSINEUX

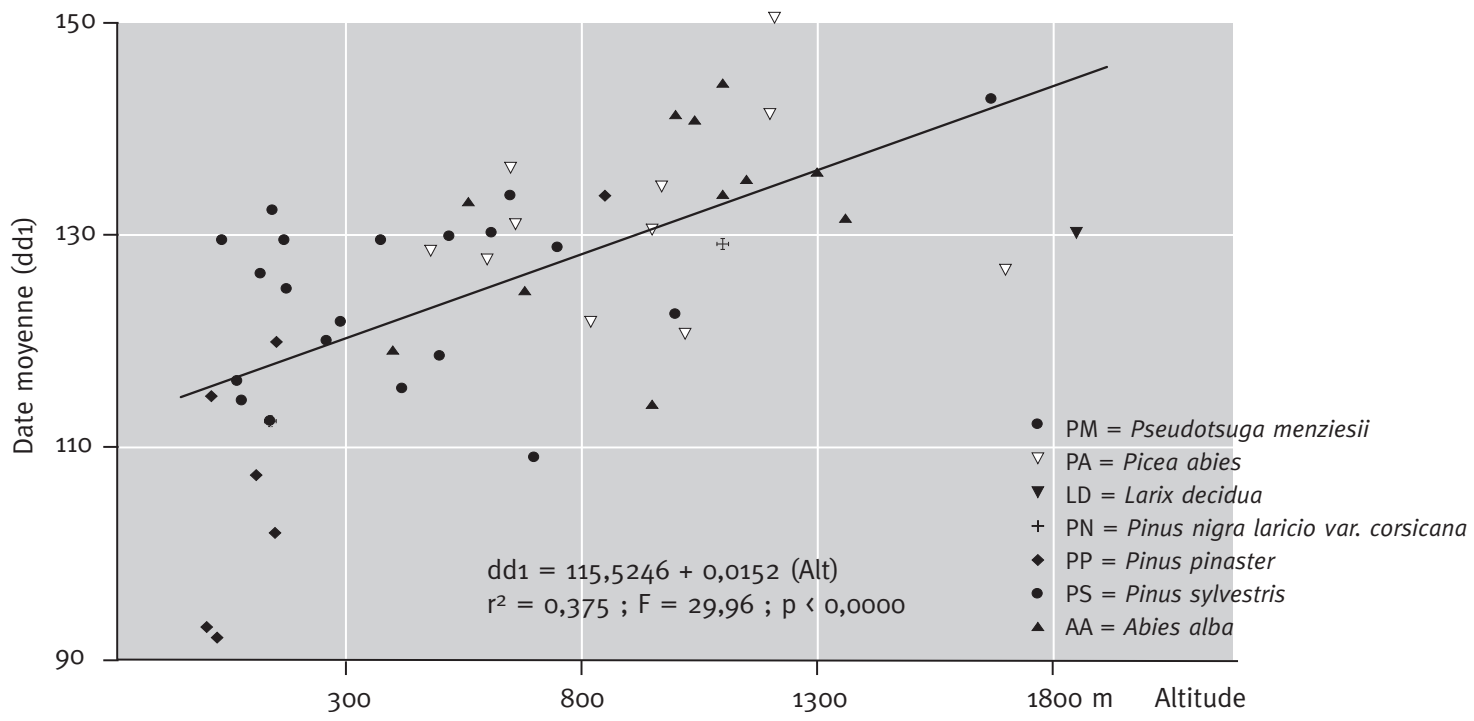


François Lebourgeois - Jean-Claude Pierrat - Vincent Perez - Christian Piedallu - Sébastien Cecchini - Erwin Ulrich

\section{Déterminisme géographique et climatique}

Un exemple d'arbre de régression simple pour la date de débourrement est présenté dans la figure 4. Dans cet exemple, le premier niveau de discrimination est l'espèce (feuillus ou résineux). Les autres niveaux sont liés à la température moyenne $\left({ }^{\circ} \mathrm{C}\right)$ ou à l'évapotranspiration (ETP Turc, mm) du début de printemps (mars-avril), à la longitude et à l'altitude (en m). Ainsi, pour les feuillus, si la température moyenne en mars-avril est inférieure à $9^{\circ} \mathrm{C}$ et l'altitude inférieure à $1285 \mathrm{~m}$, la date moyenne prédite de débourrement (dd1) correspond au jour julien 109.

FIGURE 4

\section{EXEMPLE D'ARBRE DE RÉGRESSION SIMPLE POUR LA PRÉDICTION DE LA DATE DE DÉBOURREMENT dd1 (826 données utilisées, 1997-2006)}

RMSE (Root Mean Square Error) : 9,5 jours. \% de variance expliquée : 69,2 \%.

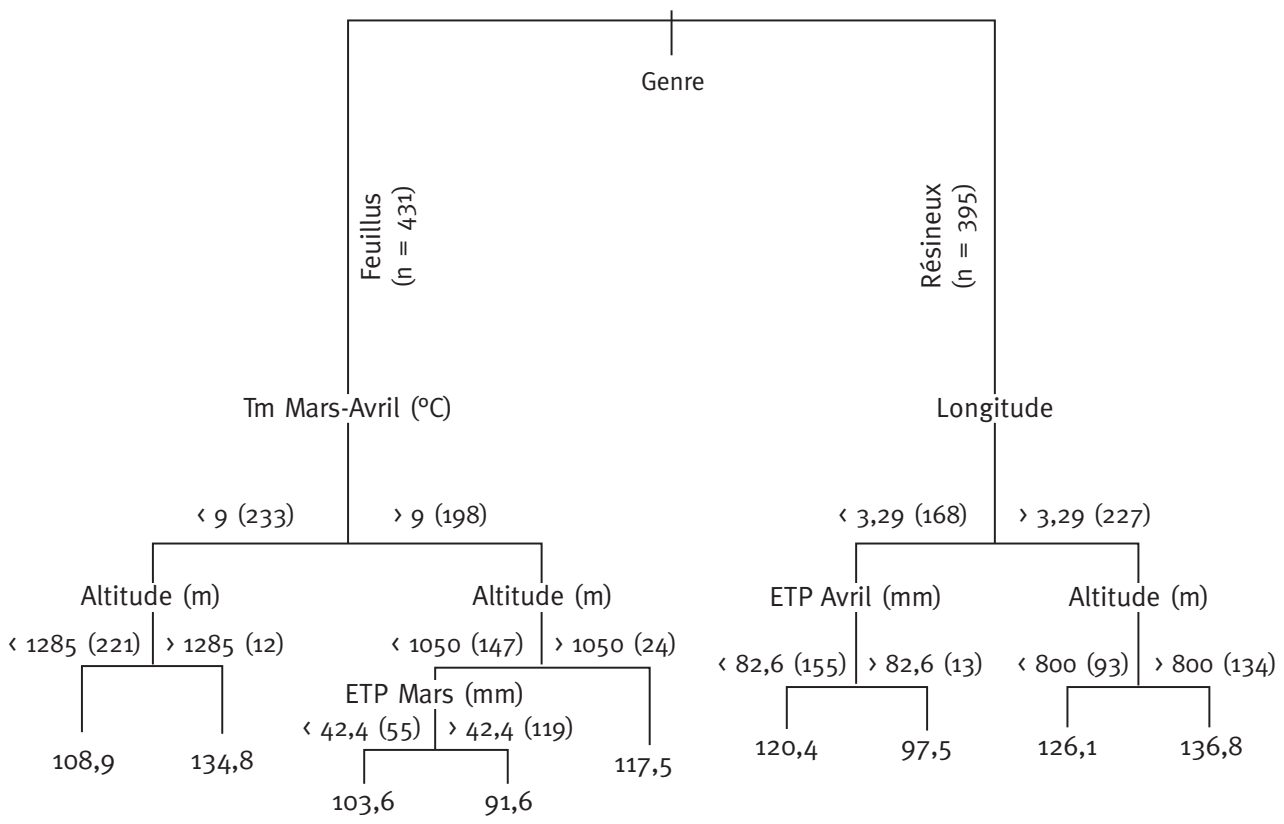

Dans la procédure complète de calcul des forêts d'arbres aléatoires, ce sont toujours les variables liées à la géographie ou au régime thermique (ETP ou température) qui sont apparues les plus discrimantes. Les précipitations ou les bilans hydriques n'apparaissent pas en tant que facteurs majeurs dans le déterminisme des phénophases. Concernant l'ETP, ce sont les estimations avec la formule de Turc qui sont ressorties majoritairement (par rapport à la formule de Thornthwaite).

Pour la date de débourrement, le genre, l'altitude, la longitude, l'évapotranspiration Turc de mars et janvier jouent le rôle le plus important parmi les 10 variables du modèle (figure 5, p. 331). L'erreur moyenne d'ajustement est de 3,7 jours et celle de la prédiction de 8,3 jours. Il est à noter que les résultats sont meilleurs en considérant dd1 plutôt que dd2.

Pour le jaunissement (feuillus exclusivement), les variables géographiques sont les plus pertinentes avec cependant un fort effet de la température moyenne du mois d'octobre. Dans ce cas, les résultats sont meilleurs avec la seconde date jj2 mais nettement moins bons que pour la 
prédiction du débourrement (figure 5 , ci-dessous). Pour la saison de végétation (jj2 - dd1), parmi les cinq premières variables, quatre sont directement liées à la géographie et une au régime thermique. Pour ces deux phénophases, le facteur distance à la mer apparaît très important. Pour le jaunissement, la distance à la Méditerranée apparaît même en seconde position avant les coordonnées géographiques.

Afin de donner une idée plus facilement interprétable des effets du régime thermique sur le débourrement, nous avons complété l'analyse précédente par une démarche plus simple fondée sur le calcul de régressions linéaires. Même si, dans les forêts d'arbres, l'ETP est apparue plus significative que la température, nous avons testé les corrélations avec la température moyenne.

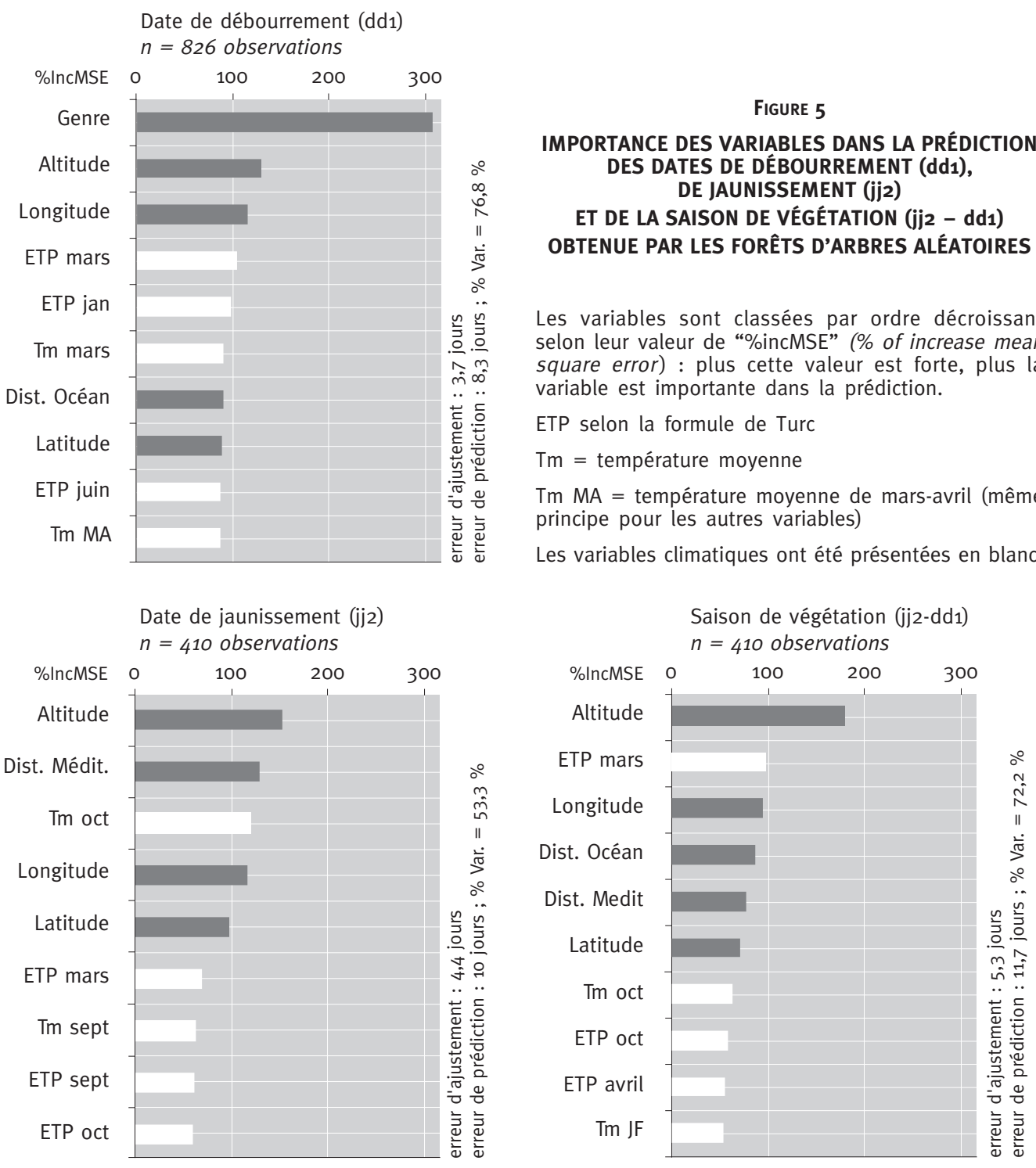


François Lebourgeois - Jean-Claude Pierrat - Vincent Perez - Christian Piedallu - Sébastien Cecchini - Erwin Ulrich

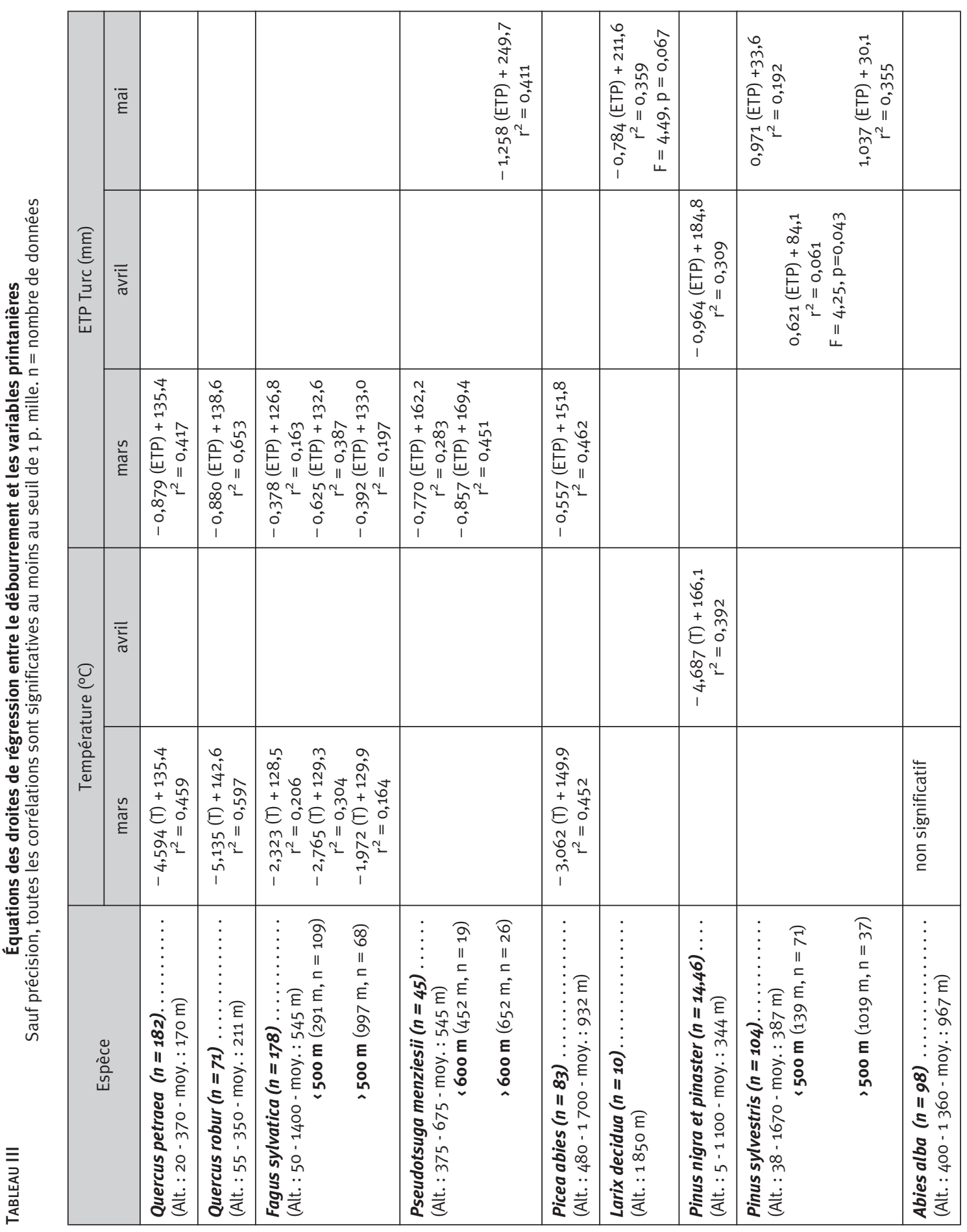


En effet, pour le gestionnaire et le praticien, les données thermiques sont généralement plus facilement accessibles que les données d'ETP qui nécessitent un calcul et l'intégration de données supplémentaires pas forcément disponibles (Lebourgeois et Piedallu, 2005). Les équations des régressions linéaires sont présentées dans le tableau III (p. 332) et la figure 6 (ci-dessous). Pour le Douglas, le Pin sylvestre et le Mélèze, seules les corrélations avec l'ETP sont significatives. Pour le Sapin, aucune corrélation significative pour les mois testés n'est ressortie. Pour les autres espèces, la corrélation avec l'ETP est apparue plus élevée dans 4 cas sur 7 . Pour les

FIGURE 6

\section{RELATION ENTRE LA DATE DE DÉBOURREMENT (dd1) ET LES CONDITIONS THERMIQUES PRINTANIÈRES}

Les équations des droites sont présentées dans le tableau III (ci-contre).

Pour le Hêtre et le Pin sylvestre, deux altitudes ont été considérées (> ou < $500 \mathrm{~m}$ ).
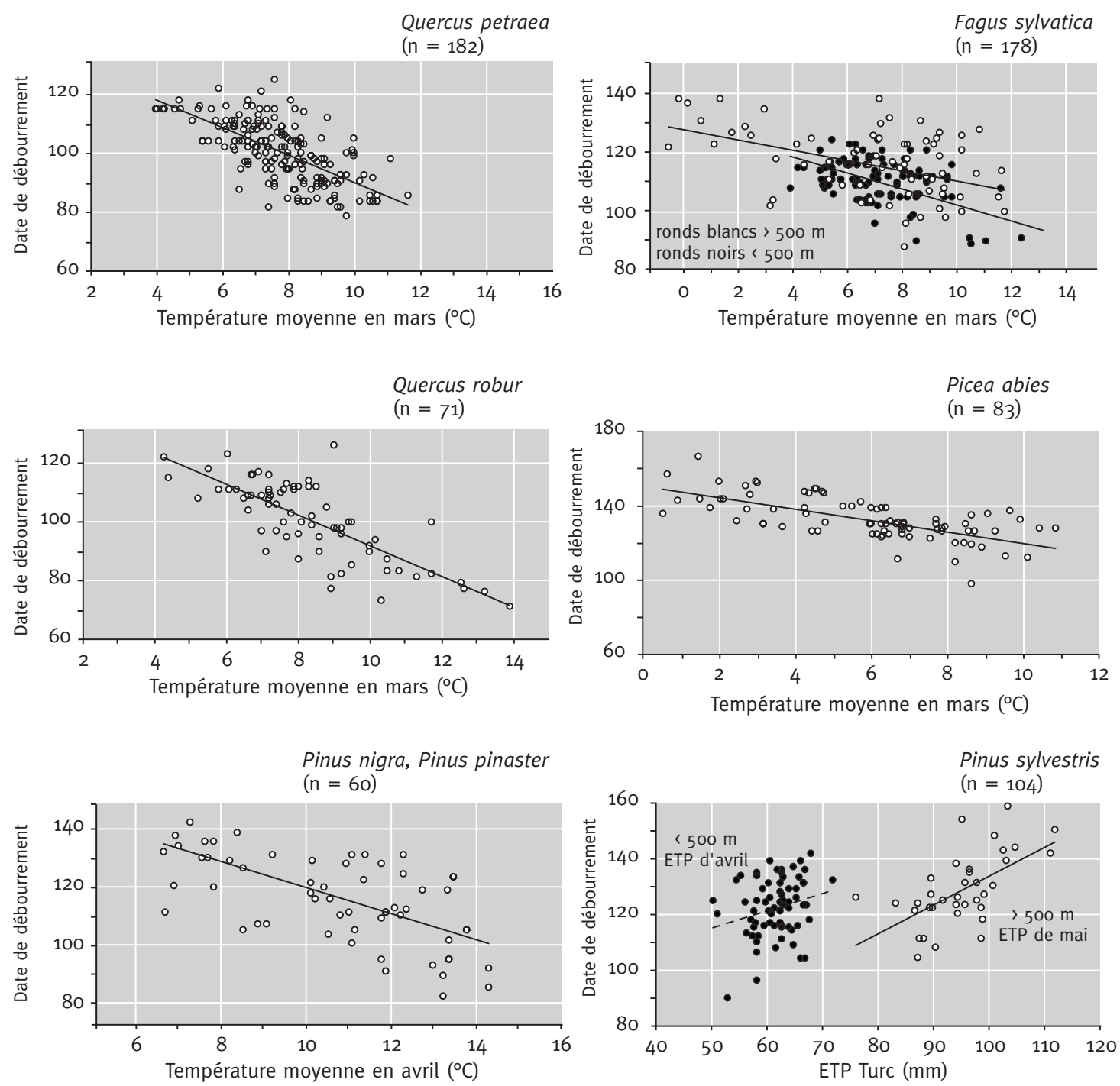
Chênes, le Hêtre et le Douglas en plaine ( 500 m) et l'Épicéa, les conditions thermiques de mars expliquent en moyenne $40 \%$ de la variabilité des dates de débourrement. Pour les peuplements résineux d'altitude, c'est l'ETP de mai qui intervient le plus souvent. Pour les feuillus, la plus forte corrélation a été observée pour le Chêne pédonculé et la moins forte pour le Hêtre. Finalement, selon les droites de régression, une augmentation de l'ETP en mars de $10 \mathrm{~mm}$ se traduit par une avancée du débourrement entre 4 et 9 jours (moyenne : 6,8 jours). Concernant la température, une augmentation de $1{ }^{\circ} \mathrm{C}$ pendant cette période se traduit par une précocité de 2 à 5 jours environ.

Concernant l'effet de la température d'octobre sur le jaunissement des feuillus, les équations des régressions simples montrent un jaunissement plus tardif de 2,2 (Hêtre), 2,4 (Chêne sessile) et 3,9 (Chêne pédonculé) jours par degré supplémentaire en octobre. Les coefficients de corrélation sont moins élevés que pour le débourrement et varient respectivement de 12,2 \%, 18,4 \% et 40,6 \% (données non montrées). Enfin, pour la saison de végétation, une augmentation de $10 \mathrm{~mm}$ de l'ETP mars se traduit par un rallongement de 5,4 (Hêtre), 10,7 (Chêne sessile) et 16,3 (Chêne pédonculé) jours. Les corrélations varient de $10 \%, 26,9 \%$ et $66,8 \%$ pour les trois espèces. Pour la température, les augmentations par degré en mars sont de 3,9 (Hêtre), 5,4 (Chêne sessile) et 8,9 (Chêne pédonculé) jours (coefficients de corrélation : $16,4 \%, 27,3 \%$ et $53,8 \%$ ).
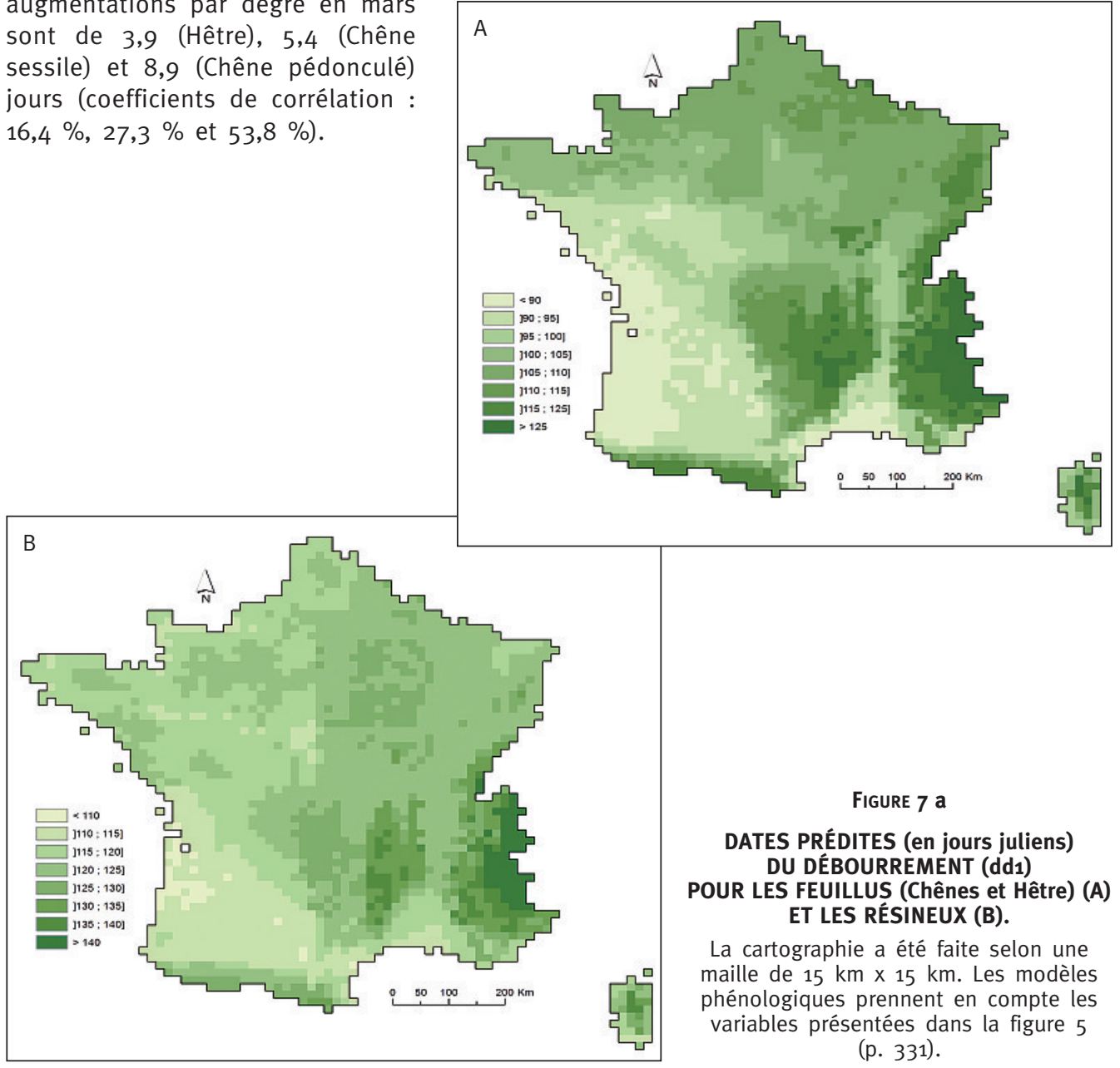

FIGURE 7 a

DATES PRÉDITES (en jours juliens) DU DÉBOURREMENT (dd1) POUR LES FEUILLUS (Chênes et Hêtre) (A) ET LES RÉSINEUX (B).

La cartographie a été faite selon une maille de $15 \mathrm{~km} \times 15 \mathrm{~km}$. Les modèles phénologiques prennent en compte les variables présentées dans la figure 5 (p. 331). 


\section{Cartographie à l'échelle de la France}

Les modèles ont été utilisés pour générer les cartes des dates moyennes des phénophases sur l'ensemble du territoire (Garzon et al., 2006 ; Iverson et Prasad, 1998). Pour cette cartographie, les variables ressortant dans les modèles ont été calculées spatialement à partir d'un système d'information géographique ou extraites de différentes bases de données. Les altitudes sont issues du modèle numérique de terrain de l'Institut géographique national. Les données climatiques mensuelles spatialisées sur la période étudiée n'étant pas facilement accessibles pour le territoire national, nous avons utilisé les bases climatiques européennes. De 1997 à 2000, les paramètres ont été extraits de la base de données du centre européen de recherche Tyndall sur les changements climatiques. Il s'agit de données observées qui sont par ailleurs disponibles sur la période 1901-2000 (http://www.cru.uea.ac.uk/) (New et al., 1999, 2000 ; New et al., 2002). Pour la période 2001-2006, les données ont été estimées à partir du modèle global $\mathrm{HadCM}_{3}$ et du scénario de changement climatique A2 (Mitchell et al., 2004). Afin d'évaluer la fiabilité de ces estimations spatiales, ces données ont été comparées aux relevés des stations Météo-France utilisés pour cette étude. Ainsi, pour l'ETP Turc de mars, la différence entre les calculs effectués sur les stations ponctuelles MétéoFrance et ceux faits à partir des données spatialisées est inférieure à $10 \mathrm{~mm}$ dans $82 \%$ des cas (différence moyenne pour la période 1997$2006=2,3 \mathrm{~mm}$ ).
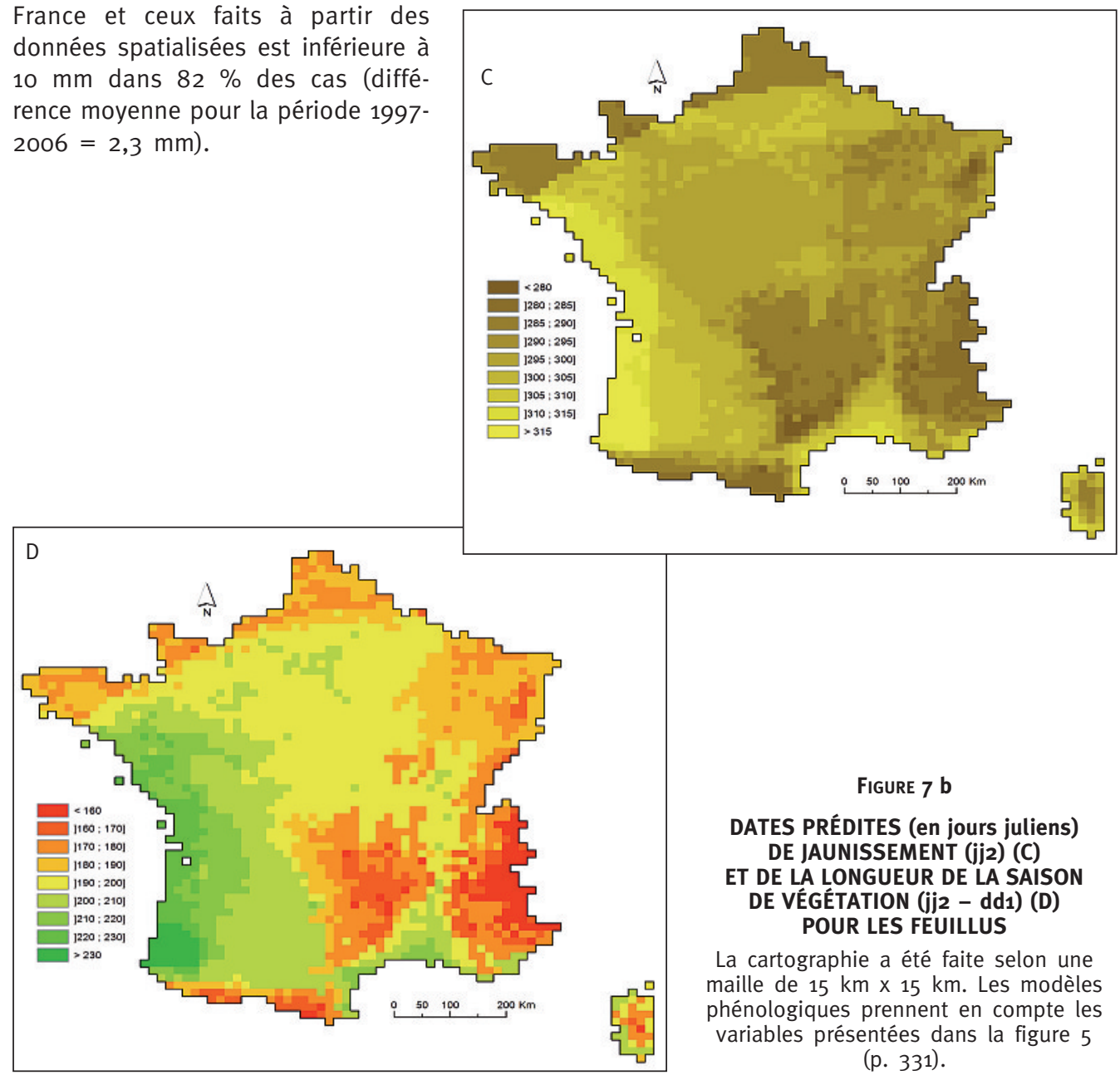

Figure 7 b

DATES PRÉDITES (en jours juliens) DE JAUNISSEMENT (jj2) (C)

ET DE LA LONGUEUR DE LA SAISON DE VÉGÉTATION (jj2 - dd1) (D) POUR LES FEUILLUS

La cartographie a été faite selon une maille de $15 \mathrm{~km} \times 15 \mathrm{~km}$. Les modèles phénologiques prennent en compte les variables présentées dans la figure 5 (p. 331). 
François Lebourgeois - Jean-Claude Pierrat - Vincent Perez - Christian Piedallu - Sébastien Cecchini - Erwin Ulrich

Les mêmes comparaisons ont été faites pour les autres paramètres avec des résultats similaires. Ainsi, même si l'utilisation de données spatialisées nationales semblait a priori plus valide, il apparaît que ces données européennes sont tout à fait utilisables pour notre étude. Étant donné que les données climatiques spatialisées correspondent à un maillage de $15 \mathrm{~km} \times 15 \mathrm{~km}$, les cartes phénologiques ont été élaborées sur l'ensemble de la France avec cette même résolution. Les cartes des dates moyennes de débourrement, de jaunissement ainsi que la longueur de saison de végétation sont présentées dans la figure 7 (p. 334-335).

\section{DISCUSSION ET CONCLUSIONS}

Les résultats présentés ici confirment les tendances déjà observées dans un article précédent (Lebourgeois et al., 2002) quant à la forte variabilité entre espèces. Ils illustrent également le rôle majeur du site (à travers les données géographiques) et des régimes thermiques printanier et automnal dans le cycle de développement des espèces forestières. Ces résultats ne sont pas nouveaux étant donné que des études précédentes menées en Europe ont déjà illustré l'importance de ces facteurs (Rötzer et Chmielewski, 2001). Cependant, aucune analyse sur des observations forestières à cette échelle spatiale n'avait encore été menée en France. De plus, même si les facteurs expliquant le mieux le déterminisme des phénophases sont cohérents avec les connaissances actuelles (Chuine et Cour, 1999 ; Chuine et Beaubien, 2001), cette étude a permis de classer leur importance respective en contexte tempéré français et de cartographier les dates moyennes prédites de différentes phases du développement foliaire.

Le genre et l'espèce apparaissent bien évidemment être les facteurs explicatifs premiers de la variabilité des phénophases. Pour les feuillus, des différences notables sont apparues entre les Chênes et le Hêtre avec un déterminisme climatique qui semble moins marqué pour ce dernier (voir figure 6, p. 333). Bien que ce résultat rejoigne des travaux précédents (Soudani et al., 2008), il est possible que la différence de répartition des sites associée à des différenciations génétiques entre des populations expliquent ces comportements. De très nombreuses études ont montré que la phénologie est un des traits les plus sélectifs qui reflètent fortement les adaptations aux conditions écologiques locales (résistance au froid, aux ravageurs) (Chmura, 2006 ; Chmura et Rozkowski, 2002 ; Chuine et al., 2000 ; Ducousso et al., 1996 ; Morin et al., 2007 ; Oleksyn et al., 1992 ; Oleksyn et al., 1998 ; Parmesan, 2007). En France, Ducousso et al. (1996) ont montré que les Chênes sessiles des hautes latitudes débourraient plus tardivement que les populations issues des latitudes plus méridionales (entre $44^{\circ}$ et $56^{\circ} \mathrm{N}$ ). Pour le Hêtre, on peut également penser que la prise en compte de facteurs comme le rayonnement global ou la durée d'insolation pourrait permettre d'améliorer les prédictions (Kramer, 1994 ; Kramer, 1995). Malheureusement, cette hypothèse n'est pas testable à vaste échelle en raison du manque de données disponibles. Bien que les conséquences du réchauffement climatique soient encore très largement inconnues, cette différence de comportement pourrait avoir des conséquences importantes à travers les gelées tardives (Dittmar et al., 2006) ou en modifiant les bilans de carbone et donc la croissance des essences (Davi et al., 2005 ; Dufrêne et al., 2005).

Dans la modélisation, l'effet direct des facteurs thermiques arrive en "second plan" après les facteurs topo-géographiques. Ces derniers intégrant déjà fortement les gradients thermique et radiatif à vaste échelle, il n'est pas surprenant que les effets directs apparaissent en second dans les variables retenues dans le modèle. Nos résultats corroborent des études précédentes (Menzel et al., 2005 ; Scheifinger et al., 2002) et les effets des facteurs géographiques sont du même ordre que ceux donnés dans ces travaux. Ainsi, en Europe centrale et de l'Est, les analyses menées sur différentes espèces et phénophases (floraison, feuillaison...) estiment un retard des 
phases printanières compris entre 0,4 et 1,6 jour par $100 \mathrm{~km}$ et une avancée des phases automnales de o,8 jour par $100 \mathrm{~km}$ dans le sens ouest-est (Rötzer et Chmielewski, 2001). Les estimations fondées sur des images satellitaires sont de l'ordre de 2 jours par degré de latitude en Amérique du Nord, en Europe et en Asie (Zhang et al., 2004). Les effets de l'altitude sont du même ordre de grandeur que les données sur Acer saccharum (2,7 jours) (Richardson et al., 2006) ou encore sur Fagus sylvatica en Allemagne (1,6-3,9 jours; 400 à $1000 \mathrm{~m}$ ) (Dittmar et Elling, 2006). Dans notre étude, la prise en compte des variables liées à la distance à la mer a amélioré les prédictions pour le jaunissement et, par conséquent, pour la saison de végétation. L'explication n'est pas évidente (effet de l'amplitude thermique, de la durée d'insolation ?) et devra faire l'objet d'analyses plus poussées. Bien qu'arrivant en second plan, les effets thermiques sont néanmoins très significatifs. Ainsi, les conditions printanières centrées sur le mois de mars et celles automnales centrées sur le mois d'octobre expliquent les dates de débourrement et de jaunissement ainsi que la longueur de la saison de végétation. Notre étude confirme donc le fort contrôle thermique des phénophases. Des corrélations linéaires entre le régime thermique de mars à mai et le débourrement ou la floraison ont été observées pour un grand nombre d'espèces aussi bien en climat tempéré que tropical (Borchert et al., 2005 ; Hänninen et Kramer, 2007 ; Holopainen et al., 2006 ; Scheifinger et al., 2002 ; Wesolowski et Rowinski, 2006). Pour la grande majorité des espèces, la variabilité des températures de fin d'hiver ou de début de printemps représente le facteur atmosphérique majeur pour le développement saisonnier (Beaubien et Freeland, 2000 ; Scheifinger et al., 2002). Dans notre cas, elle explique entre $16 \%$ et $65 \%$ des dates de débourrement (voir tableau III, p. 332). L'avance de 2 à 5 jours par degré supplémentaire en fin d'hiver-début du printemps est en accord avec des études précédentes en Europe (Chmielewski et Rötzer, 2001 ; Menzel et Fabian, 1999) ou en Amérique du Nord (Borchert et al., 2005). En Allemagne, une augmentation de la température moyenne de $1{ }^{\circ} \mathrm{C}$ entre février et avril se traduit par un rallongement de la saison et une floraison plus précoce d'environ 5 jours pour Prunus avium et Malus domestica (Chmielewski et al., 2004). L'originalité de notre étude est d'illustrer le fort effet de l'ETP Turc de mars. Cet effet n'a pas été mentionné dans les études précédentes peut-être en raison de la moindre accessibilité à ce paramètre climatique qui nécessite un calcul préalable. La plus grande efficacité de ce paramètre, par rapport à la seule température ou à la formule de l'ETP Thornthwaite, est que le calcul intégre le rayonnement global (Lebourgeois et Piedallu, 2005), paramètre dont certaines études ont montré l'importance pour prédire le débourrement (Kramer, 1994). Cette étude apporte donc aussi une nouvelle validation quant à l'intérêt d'utilisation de ce paramètre climatique en écologie (Pinto et al., 2007).

Les erreurs des ajustements de nos modèles (entre 4 et 5 jours) et de prédictions (entre 8 et 12 jours selon la variable considérée) sont cohérentes avec des études précédentes plus complexes prenant en compte des sommes de températures et des effets de seuils dans les processus de modélisation. Ainsi, en Allemagne du Sud, le débourrement du Hêtre et du Chêne pédonculé a été évalué avec une erreur de 5 jours environ (Rötzer et al., 2004). Dans une autre étude, cette erreur a varié de 4,8 à 7,5 pour le débourrement du Hêtre, de l'Épicéa, des Chênes et des Pins (Cannell et Smith, 1983 ; Kramer, 1994). Il est important de rappeler que, dans le réseau, la précision des observations étant de 5 à 7 jours, il n'est de toute façon pas envisageable d'obtenir des prédictions plus précises par modélisation. Les modèles ont néanmoins permis de cartographier à vaste échelle les dates moyennes de différentes phénophases ; dates valides sur la période récente (calibration 1997-2006). Étant donné que les modèles intégrent certains paramètres climatiques et que nous disposons maintenant de données spatialisées anciennes et des prédictions futures, une analyse rétrospective et prospective pourra être envisagée à court terme. Couplée à une analyse détaillée des observations contenues dans la base en cours de création (groupe de recherche, système d'information phénologique pour la gestion 
et l'étude des changements climatiques, http://gdr.obs-saisons.fr/), ces études devraient apporter, dans un avenir proche, des éléments d'information sur des éventuelles dérives des "dates phénologiques" en France. Associés aux études en cours sur les niches (actuelles et futures) des essences et sur leurs potentialités, ces travaux devraient permettre d'améliorer encore nos connaissances sur les traits de vie des espèces et de "mieux" appréhender les changements futurs. Cependant, même si les modèles élaborés sont statistiquement tout à fait valides, nous sommes bien conscients que la non-homogénéité de répartition des sites à travers la France peut poser quelques problèmes (effet de la longitude chez les Chênes et pas chez le Hêtre par exemple). Avec le jeu de données actuellement disponible, il n'est pas possible de cartographier espèce par espèce à l'échelle du territoire. Les cartes ont été élaborées pour les groupes "feuillus" (Hêtre et Chênes) et résineux en considérant également que ces espèces pouvaient être présentes partout. Par exemple, dans les Alpes internes, la place des feuillus (notamment des Chênes) est très réduite mais la carte actuelle donne néanmoins une valeur de débourrement (voir figure $7 \mathrm{~A}$, p. 334). Dans le futur, la carte pourrait être améliorée en considérant conjointement la présence réelle (ou future ?) des espèces et leurs phénophases.

In fine, cette étude a permis pour la première fois en France de dresser un bilan des "dates phénologiques" pour des espèces forestières, de les modéliser et de les cartographier. Les analyses futures devraient apporter des éléments d'information très importants sur les conséquences possibles du réchauffement climatique sur ce trait de vie hautement sélectif.

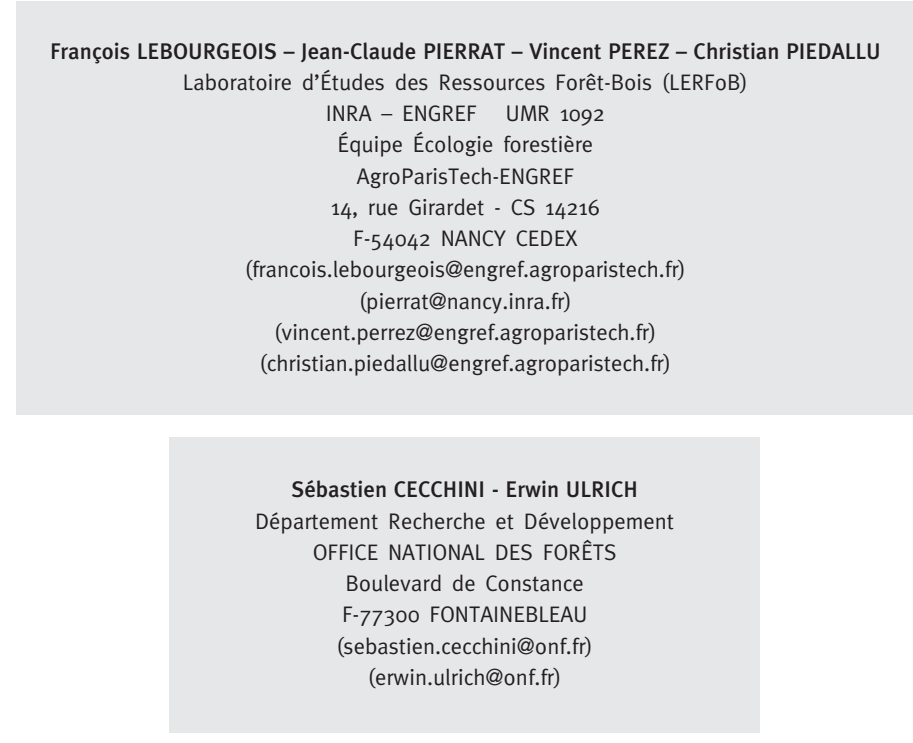

\section{Remerciements}

Les auteurs remercient l'ensemble des correspondants locaux du réseau RENECOFOR pour leur travail efficace dans la récolte des données ainsi que les lecteurs-arbitres pour leurs remarques constructives sur le manuscrit. 


\section{Les forêts d'arbres aléatoires : une technique récente d'analyse de données}

Les méthodes des forêts d'arbres aléatoires sont capables de prendre en compte les interactions multiples entre tous les régresseurs, aussi bien qualitatifs que quantitatifs, et les relations non linéaires entre ceux-ci. Ces techniques ne nécessitent pas l'hypothèse de normalité des régresseurs et, surtout, peuvent être utilisées même quand leur nombre est très supérieur à celui des observations sans que le modèle soit "surparamétré" (Berk, 2006 ; Breiman, 2001b ; Vayssières et al., 2000). Le but est de partager un échantillon de données $X$ de façon progressive en $q$ classes, $q$ n'étant pas fixé a priori, à l'aide de règles binaires et de visualiser les résultats à l'aide d'un arbre. La première séparation (= nœud) correspond au régresseur qui discrimine le plus la variabilité des observations initiales. Les deux branches de chaque nœud définissent le seuil ou la nature de discrimination du niveau hiérarchique (voir figure 4, p. 330). La procédure s'arrête quand le nombre de données devient trop faible pour définir des nœuds ou que toute l'hétérogénéité des données initiales a été prise en compte dans les différents niveaux hiérarchiques. Pour calculer les différents niveaux hiérarchiques, on utilise des fonctions d'hétérogénéité dérivées de l'entropie de Shannon et de l'indice d'inégalité de Gini (Ghattas, 1999). Dans ses travaux, Breiman a montré qu'une légère modification de l'échantillon initial $\boldsymbol{X}$ (appelé aussi échantillon d'apprentissage) pouvait modifier fortement l'arbre. Ainsi, la procédure finale consiste à stabiliser les résultats par agrégation d'arbres élémentaires construits sur $n_{\text {tree }}$ échantillons tirés au hasard dans l'ensemble des données initiales ( $n_{\text {tree }}$ échantillons "bootstrap" ou de calibration). On parle alors de "forêts d'arbres aléatoires". Dans notre étude, $n_{\text {tree }}$ a été fixé à 8000 , ce qui est largement suffisant pour assurer une très bonne stabilité des résultats (Liaw et Wiener, 2002). On peut également fixer le nombre de régresseurs maximal à prendre en compte pour discriminer chaque niveau $\left(m_{t r y}\right)$ (Peters et al., 2007). Ce paramètre peut être ajusté progressivement de façon à obtenir les meilleures prédictions. Dans notre étude, une dizaine de variables environnementales étant apparues comme particulièrement explicatives, $m_{t r y}$ a été fixé à 5. La procédure utilisée permet également de réaliser une validation croisée des prédictions. En effet, pour chaque arbre, on peut prédire les données qui n'ont pas été prises en compte dans l'échantillon de calibration en utilisant l'arbre construit sur ces données de calibration. On obtient alors un jeu de données de vérification ("out-of-bag" data) ainsi que les prédictions du facteur étudié (“OOB predictions"). L'importance de chacun des régresseurs est également testée en regardant comment l'erreur de prédiction du facteur étudié augmente quand la valeur du régresseur obtenue sur les données de vérification est permutée aléatoirement alors que tous les autres régresseurs restent inchangés. Plus le régresseur a du poids, plus une permutation aléatoire dans le jeu de données doit entraîner des erreurs de prédiction fortes. Au final, différents paramètres statistiques permettent de juger de la qualité des résultats : la qualité de l'ajustement du modèle (RMSE) et des prédictions (RMSE oob), la variance prédite (\% Var) et le poids de chaque régresseur (\% IncMSE) (voir figure 5, p. 331).

\section{BIBLIOGRAPHIE}

AHAS (R.), JAAGUS (J.), AASA (A.). - The phenological calendar of Estonia and its correlation with mean air temperature. - International Journal of Biometeorology, vol. 44, $\mathrm{n}^{\circ} 4$, 2000, pp. 159-166.

AHAS (R.), AASA (A.), MENZEL (A.), FEDOTOVA (G.), SCHEIFINGER (H.). - Changes in european spring phenology. - International Journal of Climatology, vol. 22, 2002, pp. 1727-1738. 
François Lebourgeois - Jean-Claude Pierrat - Vincent Perez - Christian Piedallu - Sébastien Cecchini - Erwin Ulrich

BEAUBIEN (E.G.), FREELAND (H.J.). - Spring phenology trends in Alberta, Canada: links to ocean temperature. - International Journal of Biometeorology, vol. 44, $\mathrm{n}^{\circ} 2$, 2000, pp. 53-59.

BERK (R.A.). - An introduction to ensemble methods for data analysis. - Sociological Methods \& Research, vol. 34, $\mathrm{n}^{\circ}$ 3, 2006, pp. 263-295.

BORCHERT (R.), ROBERTSON (K.), SCHWARTZ (M.D.), WILLIAMS-LINERA (G.). - Phenology of temperate trees in tropical climates. - International Journal of Biometeorology, vol. 50, $\mathrm{n}^{\circ}$ 1, 2005, pp. 57-65.

BREIMAN (L.). - Bagging predictors. - Machine Learning, vol. 24, 1996, pp. 123-140.

BREIMAN (L.). - RandomForests. - Machine Learning, vol. 45, 2001a, pp. 5-32.

BREIMAN (L.). - Statistical modeling: The two cultures. - Statistical Science, vol. 16, n 3, 2001b, pp. 199-215.

CANNELL (M.G.R.), SMITH (R.I.). - Thermal time, chill days and prediction of budburst in Picea sitchensis. Journal of Applied Ecology, vol. 20, 1983, pp. 951-963.

CHEN (X.Q.), HU (B.), YU (R.). - Spatial and temporal variation of phenological growing season and climate change impacts in temperate eastern China. - Global Change Biology, vol. 11, n 7, 2005, pp. 1118-1130.

CHMIELEWSKI (F.M.), RÖTZER (T.). - Response of tree phenology to climate change across Europe. - Agricultural and Forest Meteorology, vol. 108, $\mathrm{n}^{\circ}$ 2, 2001, pp. 101-112.

CHMIELEWSKI (F.M.), MULLER (A.), BRUNS (E.). - Climate changes and trends in phenology of fruit trees and field crops in Germany, 1961-2000. - Agricultural and Forest Meteorology, vol. 121, n 1-2, 2004, pp. 69-78.

CHMURA (D.J.). - Phenology differs among Norway spruce populations in relation to local variation in altitude of maternal stands in the Beskidy Mountains. - New Forests, vol. 32, $\mathrm{n}^{\circ}$ 1, 2006, pp. 21-31.

CHMURA (D.J.), ROZKOWSKI (R.). - Variability of beech provenances in spring and autumn phenology. Silvae Genetica, vol. 51, $\mathrm{n}^{\circ}$ 2-3, 2002, pp. 123-127.

CHUINE (I.), COUR (P.). - Climatic determinants of budburst seasonnality in four temperate-zone tree species. - New Phytologist, vol. 143, 1999, pp. 339-349.

CHUINE (I.), BEAUBIEN (E.G.). - Phenology is a major determinant of tree species range. - Ecology Letters, vol. $4, \mathrm{n}^{\circ}$ 5, 2001, pp. 500-510.

CHUINE (I.), BELMONTE (J.), MIGNOT (A.). - A modelling analysis of the genetic variation of phenology between tree populations. - Journal of Ecology, vol. 88, $n^{\circ} 4$, 2000, pp. 561-570.

CHURKINA (G.), SCHIMEL (D.), BRASWELL (B.H.), XIAO (X.). - Spatial analysis of growing season length control over net ecosystem exchange. - Global Change Biology, vol. 11, 2005, pp. 1777-1787.

DAVI (H.), DUfRÊNE (E.), GRANIER (A.), LE DANTEC (V.), BARBAROUX (C.), FRANÇOIS (C.), BRÉdA (N.). Modelling carbon and water cycles in a beech forest. Part II: Validation of the main processes from organ to stand scale. - Ecological Modelling, vol. 185, n²-4, 2005, pp. 387-405.

DITTMAR (C.), ELLING (W.). - Phenological phases of common beech (Fagus sylvatica L.) and their dependence on region and altitude in Southern Germany. - European Journal of Forest Research, vol. 125, $\mathrm{n}^{\circ}$ 2, 2006, pp. 181-188.

DITTMAR (C.), FRICKE (W.), ELLING (W.). - Impact of late frost events on radial growth of common beech (Fagus sylvatica L.) in Southern Germany. - European Journal of Forest Research, vol. 125, $\mathrm{n}^{\circ}$ 3, 2006, pp. 249-259.

DUCOUSSO (A.), GUYON (J.-P.), KREMER (A.). - Latitudinal and altitudinal variation of bud burst in western populations of sessile oak (Quercus petraea (Matt.) Liebl.). - Annales des Sciences forestières, vol. 53, 1996, pp. 775-782.

DUFRÊNE (E.), DAVI (H.), FRANÇOIS (C.), LE MAIRE (G.), LE DANTEC (V.), GRANIER (A.). - Modelling carbon and water cycles in a beech forest. Part I: Model description and uncertainty analysis on modelled NEE. - Ecological Modelling, vol. 185, 2005, pp. 407-436.

ESTRELLA (N.), SPARKS (T.), MENZEL (A.). - Trends and temperature response in the phenology of crops in Germany. - Global Change Biology, vol. 13, 2007, pp. 1737-1747.

GARZON (M.B.), BLAZEK (R.), NETELER (M.), DE DIOS (R.S.), OLLERO (H.S.), FURLANELLO (C.). - Predicting habitat suitability with machine learning models: The potential area of Pinus sylvestris L. in the Iberian Peninsula. - Ecological Modelling, vol. 197, n 3-4, 2006, pp. 383-393.

GHATTAS (B.). - Prévision par arbres de classification. - Mathématiques et Sciences humaines, vol. 37, $\mathrm{n}^{\circ}$ 146, 1999, pp. 31-49.

GISLASON (P.O.), BENEDIKTSSON (J.A.), SVEINSSON (J.R.). - Random Forests for land cover classification. Pattern Recognition Letters, vol. 27, $\mathrm{n}^{\circ}$ 4, 2006, pp. 294-300.

HÄNNINEN (H.), KRAMER (K.). - A framework for modelling the annual cycle of trees in boreal and temperate regions. - Silva Fennica, vol. 41, $\mathrm{n}^{\circ} 1,2007$, pp. 167-205. 
HOLOPAINEN (J.), HELAMA (S.), TIMONEN (M.). - Plant phenological data and tree-rings as palaeoclimate indicators in south-west Finland since AD 1750. - International Journal of Biometeorology, vol. 51, $n^{\circ} 1$, 2006, pp. 61-72.

IVERSON (L.R.), PRASAD (A.M.). - Predicting abundance of 80 tree species following climate change in the eastern United States. - Ecological Monographs, vol. 68, $\mathrm{n}^{\circ} 4$, 1998, pp. 465-485.

IVERSON (L.R.), PRASAD (A.), SCHWARTZ (M.W.). - Modeling potential future individual tree-species distributions in the eastern United States under a climate change scenario: a case study with Pinus virginiana. - Ecological Modelling, vol. 115, $\mathrm{n}^{\circ}$ 1, 1999, pp. 77-93.

KRAMER (K.). - Selecting a model to predict the onset of growth of Fagus sylvatica. - Journal of Applied Ecology, vol. 31, 1994, pp. 172-181.

KRAMER (K.), LEINONEN (I.), LOUSTAU (D.). - The importance of phenology for the evaluation of impact of climate change on growth of boreal, temperate and Mediterranean forests ecosystems: an overview. International Journal of Biometeorology, vol. 44, $\mathrm{n}^{\circ}$ 2, 2000, pp. 67-75.

KRAMER (N.). - Phenotypic plasticity of the phenology of seven European tree species in relation to climatic warming. - Plant, Cell and Environment, vol. 18, 1995, pp. 93-104.

LEBOURGEOIS (F.), DIFFERT (J.), GRANIER (A.), BRÉDA (N.), ULRICH (E.). - Premières observations phénologiques des peuplements du réseau national de suivi à long terme des écosystèmes forestiers (RENECOFOR). - Revue forestière française, vol. LIV, $\mathrm{n}^{\circ}$ 5, 2002, pp. 407-418.

LEBOURGEOIS (F.), PIEDALLU (C.). - Appréhender le niveau de sécheresse dans le cadre des études stationnelles et de la gestion forestière. Notions d'indices bioclimatiques. - Revue forestière française, vol. LVII, $\mathrm{n}^{\circ}$ 3, 2005, pp. 331-356.

$\mathrm{LI}$ (Y.). - Predicting materials properties and behavior using classification and regression trees. - Materials Science and Engineering a-Structural Materials Properties Microstructure and Processing, vol. 433, $\mathrm{n}^{\circ}$ 1-2, 2006, pp. 261-268.

LIAW (A.), WIENER (M.). - Classification and regression by randomForest. $-R$ News, vol. 2-3, $\mathrm{n}^{\circ} 12,2002$, pp. 18-22.

LINDERHOLM (H.W.). - Growing season changes in the last century. - Agricultural and Forest Meteorology, vol. $137, \mathrm{n}^{\circ} 1-2,2006, \mathrm{pp} .1-14$.

LU (P.L.), YU (Q.), LIU (J.D.), LEE (X.H.). - Advance of tree-flowering dates in response to urban climate change. - Agricultural and Forest Meteorology, vol. 138, n 1-4, 2006, pp. 120-131.

MENZEL (A.). - Trends in phenological phases in Europe between 1951 and 1996. - International Journal of Biometeorology, vol. 44, 2000, pp. 76-81.

MENZEL (A.), FABIAN (P.). - Growing season extended in Europe. - Nature, vol. 397, 1999, p. 695.

MENZEL (A.), SPARKS (T.H.), ESTRELLA (N.), ECKHARDT (S.). - 'SSW to NNE' - North Atlantic Oscillation affects the progress of seasons across Europe. - Global Change Biology, vol. 11, n 6, 2005, pp. 909-918.

MENZEL (A.), SPARKS (T.H.), ESTRELLA (N.), KOCH (E.), AASA (A.), AHA (R.), ALM-KUBLER (K.), BISSOLLI (P.), BRASLAVSKA (O.), BRIEDE (A.), CHMIELEWSKI (F.M.), CREPINSEK (Z.), CURNEL (Y.), DAHL (A.), DEFILA (C.), DONNELLY (A.), FILELLA (Y.), JATCZA (K.), MAGE (F.), MESTRE (A.), NORDLI (O.), PENUELAS (J.), PIRINEN (P.), REMISOVA (V.), SCHEIFINGER (H.), STRIZ (M.), SUSNIK (A.), VAN VLIET (A.J.H.), WIELGOLASKI (F.E.), ZACH (S.), ZUST (A.). - European phenological response to climate change matches the warming pattern. - Global Change Biology, vol. 12, n 10, 2006, pp. 1969-1976.

MITCHELL (T.D.), CARTER (T.R.), JONES (P.D.), HULME (M.), NEW (M.). - A comprehensive set of high-resolution grids of monthly climate for Europe and the globe : the observed record (1901-2000) and 13 scenarios (2001-2100). - Tyndall Centre Working Paper 55, 2004, pp. 1-25.

MORIN (X.), AMEGLIO (T.), AHAS (R.), KURZ-BESSON (C.), LANTA (V.), LEBOURGEOIS (F.), MIGLIETTA (F.), CHUINE (I.). - Variation in cold hardiness and carbohydrate concentration from dormancy induction to bud burst among provenances of three European oak species. - Tree Physiology, vol. 27, $\mathrm{n}^{\circ}$ 6, 2007 , pp. 817-825.

MURRAY (M.B.), CANNELL (G.R.), SMITH (I.). - Date of budburst of fifteen tree species in Britain following climatic warming. - Journal of Applied Ecology, vol. 26, 1989, pp. 693-700.

NEW (M.), HULME (M.), JONES (P.). - Representing twentieth-century space-time climate variability. Part I: Development of a 1961-90 mean monthly terrestrial climatology. - Journal of Climate, vol. 12, $\mathrm{n}^{\circ}$ 3, 1999, pp. $829-856$.

NEW (M.), HULME (M.), JONES (P.). - Representing twentieth-century space-time climate variability. Part II: Development of 1901-96 monthly grids of terrestrial surface climate. - Journal of Climate, vol. 13, $\mathrm{n}^{\circ} 13$, 2000 , pp. 2217-2238. 
François Lebourgeois - Jean-Claude Pierrat - Vincent Perez - Christian Piedallu - Sébastien Cecchini - Erwin Ulrich

NEW (M.), LISTER (D.), HULME (M.), MAKIN (I.). - A high-resolution data set of surface climate over global land areas. - Climate Research, vol. 21, $\mathrm{n}^{\circ}$ 1, 2002, pp. 1-25.

OLEKSYN (J.), TJOELKER (M.G.), REICH (P.B.). - Growth and biomass partitioning of populations of European Pinus sylvestris L. under simulated $50^{\circ}$ and $60^{\circ} \mathrm{N}$ daylengths : evidence for photoperiodic ecotypes. New Phytologist, vol. 120, 1992, pp. 561-574.

OLEKSYN (J.), MODRZYNSKI (J.), TJOELKER (M.G.), ZYTKOWIAK (R.), REICH (P.B.), KAROLEWSKI (P.). - Growth and physiology of Picea abies populations from elevational transects: common garden evidence for altitudinal ecotypes and cold adaptation. - Functional Ecology, vol. 12, n 4, 1998, pp. 573-590.

PARMESAN (C.). - Influences of species, latitudes and methodologies on estimates of phenological response to global warming. - Global Change Biology, vol. 13, 2007, pp. 1860-1872.

PETERS (J.), DE BAETS (B.), VERHOEST (N.E.C.), SAMSON (R.), DEGROEVE (S.), DE BECKER (P.), HUYBRECHTS (W.). - Random forests as a tool for ecohydrological distribution modelling. - Ecological Modelling, vol. 207, $\mathrm{n}^{\circ}$ 2-4, 2007, pp. 304-318.

PINTO (P.), GÉGOUT (J.-C.), HERVÉ (J.-C.), DHÔTE (J.-F.). - Changes in environmental controls on the growth of Abies alba Mill. in the Vosges Mountains, north-eastern France, during the 2oth century. - Global Ecology and Biogeography, vol. 16, 2007, pp. 472-484.

PRASAD (A.M.), IVERSON (L.R.), LIAW (A.). - Newer classification and regression tree techniques: Bagging and random forests for ecological prediction. - Ecosystems, vol. 9, $\mathrm{n}^{\circ}$ 2, 2006, pp. 181-199.

PUdAs (E.), LePPAELAE (M.), TOLVANEN (A.), POIKOLAINEN (J.), VENAELAEINEN (A.), KUBIN (E.). - Trends in phenology of Betula pubescens across the boreal zone in Finland. - International Journal of Biometeorology, vol. 52, $\mathrm{n}^{\circ} 4,2008$, pp. 251-259.

REMM (K.). - Case-based predictions for species and habitat mapping. - Ecological Modelling, vol. 177, $n^{\circ}$ 3-4, 2004, pp. 259-281.

RICHARDSON (A.D.), BAILEY (A.S.), DENNY (E.G.), MARTIN (C.W.), O’KEEFE (J.). - Phenology of a northern hardwood forest canopy. - Global Change Biology, vol. 12, $\mathrm{n}^{\circ}$ 7, 2006, pp. 1174-1188.

RÖTZER (T.), CHMIELEWSKI (F.M.). - Phenological maps of Europe. - Climate Research, vol. 18, 2001, pp. $248-257$.

RÖTZER (T.), GROTE (R.), PRETZSCH (H.). - The timing of bud burst and its effect on tree growth. - International Journal of Biometeorology, vol. 48, 2004, pp. 109-118.

SCHEIFINGER (H.), MENZEL (A.), KOCH (E.), PETER (C.), AHAS (R.). - Atmospheric mechanisms governing the spatial and temporal variability of phenological phases in central Europe. - International Journal of Climatology, vol. 22, $\mathrm{n}^{\circ}$ 14, 2002, pp. 1739-1755.

SCHWARTZ (M.D.). - Advancing to full bloom: planning phenological research for the 21st century. - International Journal of Biometeorology, vol. 42, $\mathrm{n}^{\circ} 3,1999$, pp. 113-118.

SCHWARTZ (M.D.), AHAS (R.), AASA (A.). - Onset of spring starting earlier across the Northern Hemisphere. - Global Change Biology, vol. 12, $\mathrm{n}^{\circ}$ 2, 2006, pp. 343-351.

SOUDANI (K.), LE MAIRE (G.), DUFRÊNE (E.), FRANÇOIS (C.), DELPIERRE (N.), ULRICH (E.), CECCHINI (S.). Evaluation of the onset of green-up in temperate deciduous broadleaf forests derived from Moderate Resolution Imaging Spectroradiometer (MODIS) data. - Remote Sensing of Environment, vol. 112, $\mathrm{n}^{\circ}$, 2008, pp. 2643-2655.

SPARKS (T.H.), MENZEL (A.). - Observed changes in seasons: An overview. - International Journal of Climatology, vol. 22, $\mathrm{n}^{\circ} 14,2002$, pp. 1715-1725.

TAO (F.L.), YOKOZAWA (M.), XU (Y.L.), HAYASHI (Y.), ZHANG (Z.). - Climate changes and trends in phenology and yields of field crops in China, 1981-2000. - Agricultural and Forest Meteorology, vol. 138, $\mathrm{n}^{\circ} 1-4,2006$, pp. 82-92.

ULRICH (E.). - Le Réseau RENECOFOR : objectifs et réalisation. - Revue forestière française, vol. XLVII, $n^{\circ} 2$, 1995, pp. 107-124.

VAYSSIĖRES (M.P.), PLANT (R.E.), ALLEN-DIAZ (B.H.). - Classification trees: An alternative non-parametric approach for predicting species distributions. - Journal of Vegetation Science, vol. 11, $n^{\circ} 5,2000$, pp. 679-694.

VERBYLA (D.L.). - Classification trees: a new discrimination tool. - Canadian Journal of Forest Research, vol. $17, \mathrm{n}^{\circ}$ 9, 1987, pp. 1150-1152.

WAHEED (T.), BONNELL (R.B.), PRASHER (S.O.), PAULET (E.). - Measuring performance in precision agriculture: CART - A decision tree approach. - Agricultural Water Management, vol. 84, $\mathrm{n}^{\circ}$ 1-2, 2006, pp. 173-185. 
WALTHER (G.R.), POST (E.), CONVEY (P.), MENZEL (A.), PARMESAN (C.), BEEBEE (T.J.C.), FROMENTIN (J.M.), HOEGH-GULDBERG (O.), BAIRLEIN (F.). - Ecological responses to recent climate change. - Nature, vol. 416, $\mathrm{n}^{\circ} 6879,2002$, pp. 389-395.

WESOLOWSKI (T.), ROWINSKI (P.). - Timing of bud burst and tree-leaf development in a multispecies temperate forest. - Forest Ecology and Management, vol. 237, $\mathrm{n}^{\circ}$ 1-3, 2006, pp. 387-393.

WIELGOLASKI (F.E.). - Phenological modifications in plants by various edaphic factors. - International Journal of Biometeorology, vol. 45, $\mathrm{n}^{\circ}$ 4, 2001, pp. 196-202.

ZHANG (X.Y.), FRIEDL (M.A.), SCHAAF (C.B.), STRAHLER (A.H.). - Climate controls on vegetation phenological patterns in northern mid- and high latitudes inferred from MODIS data. - Global Change Biology, vol. 10, $\mathrm{n}^{\circ}$ 7, 2004, pp. 1133-1145.

\section{dÉTERMINISME DE LA PHÊNOLOGIE DES FORÊTS TEmPÊREEES FRANÇAISES : ÉTUDE SUR LES PEUPLEmENTS dU RÉSERU RENECOFOR [Résumé]}

La variabilité spatiale et temporelle et le déterminisme du débourrement (838 observations) et du jaunissement (449) de 103 peuplements feuillus et résineux (10 espèces) du réseau RENECOFOR ont été étudiés sur la période 1997-2006. Pour les chênaies, le débourrement commence en moyenne vers la mi-avril et le jaunissement apparaît vers la mi-octobre. Le gradient ouest-est correspond à une saison de végétation plus courte à l'est (180-190 jours contre 210-220 jours) en conséquence d'un débourrement plus tardif (retard de 2 jours par degré de longitude) et d'un jaunissement plus précoce (5-10 jours). Pour le Hêtre, la saison de végétation débute plus tard vers la fin de la troisième semaine d'avril. Le jaunissement des feuilles intervenant début octobre, la saison de végétation est en moyenne de 180 jours. Le débourrement est plus tardif pour les résineux et dépend fortement de l'altitude (retard d'environ 1,5 jour par $100 \mathrm{~m}$ ). Outre l'espèce, les dates des phénophases dépendent des facteurs topo-géographiques (longitude, altitude, distance à la mer) et des régimes printanier ou automnal (ETP Turc ou température de mars ou d'octobre selon le stade considéré). Une augmentation de l'ETP en mars de $10 \mathrm{~mm}$ se traduit par une avancée du débourrement entre 4 et 9 jours selon les espèces (moyenne : 6,8 jours). Concernant la température, une augmentation de $1^{\circ} \mathrm{C}$ pendant cette période se traduit par une précocité de 2 à 5 jours environ. Les modèles globaux permettent de prédire avec une précision de 8,10 et 12 jours les trois phases considérées (débourrement, jaunissement et longueur de la saison de végétation). Les modèles ont été utilisés pour générer les cartes des dates moyennes des phénophases sur l'ensemble du territoire. Les résultats sont discutés notamment vis-à-vis du réchauffement climatique.

\section{PHENOLOGICAL TIMING IN FRENCH TEMPERATE FORESTS - A STUDY ON STRNDS IN THE RENECOFOR NETUORK [Abstract]}

The spatial and temporal variability of bud burst (838 observations) and yellowing (449) of 103 coniferous and broadleaved stands (10 species) sampled in the Renecofor network were studied over the period 19972006. For oak stands, the growing season starts in mid-April and stops in mid-October. The growing season is shorter in eastern France as a result of later bud burst (roughly 2 days delay per degree of longitude) and earlier yellowing (5 to 10 days). The growing season for beech begins somewhat later towards the end of the third week of April. Yellowing is seen in early October, making for an average growing season duration of 180 days. Bud burst is even later for coniferous stands and mainly depends on altitude (roughly 1.5 days delay per $100 \mathrm{~m}$ ). Apart from species, modelling processes highlight the influence of topographic and geographic parameters (longitude, altitude, distance from the sea) and of spring and autumn weather conditions (Turc PET and/or mean temperature in March and October depending on the phenological stage). A 10-mm increase in PET in March advances budbreak from 4 to 9 days depending on species (mean: 6.8 days). For temperature, $\mathrm{a} 1^{\circ} \mathrm{C}$ increase in March speeds up bud burst by 2 to 5 days. The overall models predict the three phases - leaf unfolding, yellowing and duration of the growing season - within 8, 10 and 12 days respectively. The resulting equations were used to generate phenological timing maps for the whole of France. Results are discussed, in particular in the context of global warming. 